\title{
A new SAIR model on complex networks for analysing the 2019 novel coronavirus (COVID-19)
}

\author{
Congying Liu • Xiaoqun Wu • Riuwu Niu • \\ Xiuqi Wu • Ruguo Fan
}

Received: 25 April 2020 / Accepted: 17 May 2020 / Published online: 15 June 2020

(C) Springer Nature B.V. 2020

\begin{abstract}
Nowadays, the novel coronavirus (COVID19) is spreading around the world and has attracted extremely wide public attention. From the beginning of the outbreak to now, there have been many mathematical models proposed to describe the spread of the pandemic, and most of them are established with the assumption that people contact with each other in a homogeneous pattern. However, owing to the difference of individuals in reality, social contact is usually heterogeneous, and the models on homogeneous networks cannot accurately describe the outbreak. Thus, we propose a susceptible-asymptomaticinfected-removed (SAIR) model on social networks to describe the spread of COVID-19 and analyse the outbreak based on the epidemic data of Wuhan from January 24 to March 2. Then, according to the results of the simulations, we discover that the measures that can curb the spread of COVID-19 include increasing the
\end{abstract}

C. Liu $\cdot$ X. Wu $(\varangle) \cdot$ X. Wu

School of Mathematics and Statistics, Wuhan University, Hubei 430072, China

e-mail: xqwu@whu.edu.cn

X. Wu

Hubei Key Laboratory of Computational Science, Wuhan University, Hubei 430072, China

R. Niu

College of Mathematics and Statistics, Shenzhen

University, Shenzhen 518060, China

R. Fan

School of Economics and Management, Wuhan University, Hubei 430072, China recovery rate and the removed rate, cutting off connections between symptomatically infected individuals and their neighbours, and cutting off connections between hub nodes and their neighbours. The feasible measures proposed in the paper are in fair agreement with the measures that the government took to suppress the outbreak. Furthermore, effective measures should be carried out immediately, otherwise the pandemic would spread more rapidly and last longer. In addition, we use the epidemic data of Wuhan from January 24 to March 2 to analyse the outbreak in the city and explain why the number of the infected rose in the early stage of the outbreak though a total lockdown was implemented. Moreover, besides the above measures, a feasible way to curb the spread of COVID19 is to reduce the density of social networks, such as restricting mobility and decreasing in-person social contacts. This work provides a series of effective measures, which can facilitate the selection of appropriate approaches for controlling the spread of the COVID19 pandemic to mitigate its adverse impact on people's livelihood, societies and economies.

Keywords COVID-19 · social network · SAIR model $\cdot$ latent period

\section{Introduction}

In the last two decades, large-scale pandemics caused by coronaviruses have occurred three times, one of 
which was the outbreak of Severe Acute Respiratory Syndrome (SARS) in 2003 in Guangdong Province of China [1], the others were the outbreak of Middle East Respiratory Syndrome (MERS) in 2012 in Saudi Arabia and 2015 in South Korea [2,3]. Unfortunately, at the end of December 2019, a new kind of coronavirus, called COVID-19, was discovered in Wuhan, the capital of Hubei Province of China. It is reported that COVID-19 is a single-stranded RNA virus and usually causes respiratory symptoms and fever, as well as death in severe cases $[4,5]$. Later on, during investigating and tracking the infected, it is found that a part of infected people are asymptomatic, but could infect others. Besides, Wuhan has a large and highly mobile scale of population and is a transportation hub in central China. These factors made the prevention and control of COVID-19 even more tough. So, on January 23 Wuhan went into lockdown and the government took first-level public health emergency response to the outbreak.

With COVID-19 spreading around the world, there have emerged an enormous number of works about the pandemic [6-10]. Tang et al. [11] proposed a deterministic susceptible-exposed-infected-removed (SEIR) compartmental model to describe the spread of COVID-19, and used partial data obtained for the confirmed cases of COVID-19 to estimate the basic reproduction number of the disease transmission. Zhao et al. [12] modelled the epidemic time series of COVID-19 cases and estimated the transmission rate of COVID-19 via the basic reproduction number based on the data of Wuhan from January 10 to 24 . Wu et al. [13] used a SEIR compartmental model to infer the number of infections in Wuhan from December 1, 2019, to January 25, 2020, from the data from December 31 to January 28 on the number of cases exported from Wuhan internationally. Above works assume that population is mixed homogeneously. But in reality, the differences of individuals and environments lead to particular contact patterns among people; thus, models with the homogeneousness assumption do not apply to real cases. Therefore, to describe the outbreak accurately, we establish a model on a social network, where nodes represent individuals and links stand for the contacts between individuals.

Furthermore, it is found that it is practical and meaningful to study propagation of epidemics on networks [14-18]. In 2002, Newman [19] showed that a large class of the so-called SIR models of epidemic disease can be solved exactly on a wide variety of networks using a combination of mapping to percola- tion models and generating function methods. Later on, Pastor-Satorras et al. [20] modelled the spread of epidemics on scale-free networks. It was found that epidemics was prevalent on scale-free networks and the topology of networks had a great influence on the behaviours of epidemic spreading. Granell et al. [21] combined the spread of information and diseases to propose an unaware-aware-unaware-susceptibleinfected-susceptible (UAU-SIS) model on networks and revealed that information awareness prevented epidemic spreading. Wei et al. [22] modelled a cooperative spreading process over an interconnected network and found that inter-layer links promoted epidemics spreading. Existing works indicate that behaviours of epidemic spreading are not only influenced by the characteristics of the diseases, but also impacted by the underlying network structures [23-27]. As Wuhan went into lockdown from January 23, ignoring the number of medical teams dispatched to assist Wuhan, the newborn, natural deaths and deaths caused by reasons other than the coronavirus, we can assume that the total population in Wuhan is unchanged. Therefore, modelling the outbreak of COVID-19 during the lockdown on a sparse social network is reasonable. We will explore whether the measures the government took were efficient and why the number of the infected was rising in the early stage even though the government took several restrictive measures to fight against the novel coronavirus.

Motivated by above discussions, we introduce a new SAIR model on a social network to describe the spread of COVID-19 in Wuhan after January 23 and then analyse how to suppress the spread of the pandemic. Specially, by calculating the basic reproduction number, we find that there are several measures that can curb COVID-19 spreading, and these measures are in fair agreement with the measures the government took. Furthermore, based on the epidemic data of Wuhan from January 24 to March 2, we analyse the outbreak in Wuhan and find that the outbreak is gradually under control after February 13.

The paper is organized as follows. In Sect. 2, a SAIR model is established and the basic reproduction number is obtained. Next, numerical simulations are provided to verify the effectiveness of the proposed measures with the model in Sect. 3. Then, some analyses and discussions about the outbreak of COVID-19 are given in Sect. 4. Finally, conclusions are drawn in Sect. 5 


\section{Model and analysis}

As is well known, there exists asymptomatic transmission in the spread of COVID-19; thus, we propose a new SAIR model on network $G$ which consists of susceptible $(S)$, asymptomatically infected $(A)$, symptomatically infected $(I)$ and removed $(R)$ individuals, and the total number of individuals is $N$. For a susceptible individual, if he (she) is infected by contacting with infected individuals (including asymptomatically and symptomatically infected individuals), he (she) could become symptomatic or asymptomatic. For an asymptomatically infected individual, he (she) might be symptomatic after a latent period or get immunized against the virus. Define $S_{k}$ as the fraction of susceptible individuals out of the individuals with degree $k$. Similarly, $A_{k}, I_{k}$ and $R_{k}$ represent the fraction of asymptomatically infected, symptomatically infected and removed individuals with degree $k$, respectively. Obviously, $S_{k}(t), A_{k}(t), I_{k}(t), R_{k}(t) \geq 0$ and $S_{k}(t)+A_{k}(t)+I_{k}(t)+R_{k}(t)=1$. The propagation process of COVID-19 can be described by

$$
\left\{\begin{array}{l}
\frac{\mathrm{d} S_{k}(t)}{\mathrm{d} t}=-\beta_{1} k S_{k}(t) \Theta_{A}(t)-\beta_{2} k S_{k}(t) \Theta_{I}(t), \\
\frac{\mathrm{d} A_{k}(t)}{\mathrm{d} t}=\alpha\left(\beta_{1} k S_{k}(t) \Theta_{A}(t)\right. \\
\left.+\beta_{2} k S_{k}(t) \Theta_{I}(t)\right)-m A_{k}(t-\tau)-\gamma_{1} A_{k}(t), \\
\frac{\mathrm{d} I_{k}(t)}{\mathrm{d} t}=(1-\alpha)\left(\beta_{1} k S_{k}(t) \Theta_{A}(t)\right. \\
\left.+\beta_{2} k S_{k}(t) \Theta_{I}(t)\right)+m A_{k}(t-\tau)-\gamma_{2} I_{k}(t), \\
\frac{\mathrm{d} R_{k}(t)}{\mathrm{d} t}=\gamma_{1} A_{k}(t)+\gamma_{2} I_{k}(t),
\end{array}\right.
$$

where $\Theta_{A}(t)=\frac{\sum_{k^{\prime}} k^{\prime} p\left(k^{\prime}\right) A_{k^{\prime}}(t)}{\langle k\rangle} \quad$ and $\Theta_{I}(t)=\frac{\sum_{k^{\prime}} k^{\prime} p\left(k^{\prime}\right) I_{k^{\prime}}(t)}{\langle k\rangle}$. Here, $p(k)$ is the degree distribution of network $G$ and $\langle k\rangle$ is the average degree of network $G$ (Table 1).

Define $X=\left(S_{k_{1}}, \ldots, S_{k_{n}}, A_{k_{1}}, \ldots, A_{k_{n}}, I_{k_{1}}, \ldots\right.$ $\left., I_{k_{n}}, R_{k_{1}}, \ldots, R_{k_{n}}\right)$, where $\left\{k_{1}, \ldots, k_{n}\right\}$ is a set of the node degrees of network $G$. It is obvious that system (1) has a disease-free equilibrium $E^{0}=$ $(1, \ldots, 1,0, \ldots, 0)$. By the definition of next-generation matrix [28], combining with compartments $A_{k}$ and $I_{k}$ in system (1), we get $F$, the growth rate of secondary infection, and $V$, the rate of individuals removed from compartments $A_{k}$ and $I_{k}$. The expressions of $F$ and $V$
Table 1 Definition of parameters

\begin{tabular}{lc}
\hline Parameters & Definition \\
\hline$\beta_{1}$ & Transmission rate of \\
& asymptomatically \\
& infected \\
& individuals \\
$\beta_{2}$ & Transmission rate of \\
& symptomatically \\
& infected \\
& individuals \\
$\alpha$ & Rate of being \\
& asymptomatic after \\
& infected \\
& Rate of being \\
& symptomatic after \\
& latent period \\
$\gamma_{1}$ & Latent period \\
& Recovery rate of \\
& asymptomatically \\
$\gamma_{2}^{\mathrm{a}}$ & infected \\
& individuals \\
& Removed rate of \\
& symptomatically \\
& infected \\
& individuals \\
& \\
\hline &
\end{tabular}

${ }^{\text {a }}$ Here, removed rate $\gamma_{2}$ is the sum of death rate and recovery rate

are

$$
\begin{gathered}
F=\left[\begin{array}{cc}
\alpha \beta_{1} P & \alpha \beta_{2} P \\
(1-\alpha) \beta_{1} P & (1-\alpha) \beta_{2} P
\end{array}\right] \text { and } \\
V=\left[\begin{array}{cc}
\left(m+\gamma_{1}\right) I_{n} & 0 \\
-m I_{n} & \gamma_{2} I_{n}
\end{array}\right] \text {, respectively, } \\
\text { where } P=\frac{1}{\langle k\rangle}\left[\begin{array}{cccc}
k_{1}^{2} p\left(k_{1}\right) & k_{1} k_{2} p\left(k_{2}\right) & \ldots & k_{1} k_{n} p\left(k_{n}\right) \\
k_{2} k_{1} p\left(k_{1}\right) & k_{2}^{2} p\left(k_{2}\right) & \ldots & k_{2} k_{n} p\left(k_{n}\right) \\
\vdots & \vdots & \ddots & \vdots \\
k_{n} k_{1} p\left(k_{1}\right) & k_{n} k_{2} p\left(k_{2}\right) & \ldots & k_{n}^{2} p\left(k_{n}\right)
\end{array}\right]
\end{gathered}
$$

and $I_{n}$ is the $n \times n$ identity matrix.

Then, the basic reproduction number is $R_{0}=$ $\rho\left(F V^{-1}\right)$, where $\rho(\cdot)$ is the spectrum radius of a matrix. Thus, we obtain

$$
\begin{aligned}
F V^{-1} & =\left[\begin{array}{cc}
\alpha \beta_{1} P & \alpha \beta_{2} P \\
(1-\alpha) \beta_{1} P & (1-\alpha) \beta_{2} P
\end{array}\right] \\
& {\left[\begin{array}{cc}
\frac{1}{m+\gamma_{1}} I_{n} & 0 \\
\frac{m}{\left(m+\gamma_{1}\right) \gamma_{2}} I_{n} & \frac{1}{\gamma_{2}} I_{n}
\end{array}\right] } \\
& =\left[\begin{array}{cc}
\left(\frac{\alpha \beta_{1}}{m+\gamma_{1}}+\frac{m \alpha \beta_{2}}{\left(m+\gamma_{1}\right) \gamma_{2}}\right) P & \frac{\alpha \beta_{2}}{\gamma_{2}} P \\
\left(\frac{(1-\alpha) \beta_{1}}{m+\gamma_{1}}+\frac{m(1-\alpha) \beta_{2}}{\left(m+\gamma_{1}\right) \gamma_{2}}\right) P & \frac{(1-\alpha) \beta_{2}}{\gamma_{2}} P
\end{array}\right] .
\end{aligned}
$$


As $\operatorname{rank}\left(F V^{-1}\right)=\operatorname{rank}(P)$, there is

$$
\begin{aligned}
\rho\left(F V^{-1}\right) & =\operatorname{trace}\left(\left(\frac{\alpha \beta_{1}}{m+\gamma_{1}}+\frac{m \alpha \beta_{2}}{\left(m+\gamma_{1}\right) \gamma_{2}}\right.\right. \\
& \left.\left.+\frac{(1-\alpha) \beta_{2}}{\gamma_{2}}\right) P\right) \\
& =\left(\frac{\alpha \beta_{1}}{m+\gamma_{1}}+\frac{m \alpha \beta_{2}}{\left(m+\gamma_{1}\right) \gamma_{2}}\right. \\
& \left.+\frac{(1-\alpha) \beta_{2}}{\gamma_{2}}\right) \operatorname{trace}(P),
\end{aligned}
$$

where $\operatorname{rank}(\cdot)$ is the rank of a matrix and trace $(\cdot)$ is the sum of the diagonal elements of a matrix. As $\operatorname{trace}(P)=\frac{\sum_{i=1}^{n} k_{i}^{2} p\left(k_{i}\right)}{\langle k\rangle}=\frac{\left\langle k^{2}\right\rangle}{\langle k\rangle}$, the basic reproduction number $R_{0}$ is

$$
R_{0}=\left(\frac{\alpha \beta_{1}}{m+\gamma_{1}}+\frac{m \alpha \beta_{2}}{\left(m+\gamma_{1}\right) \gamma_{2}}+\frac{(1-\alpha) \beta_{2}}{\gamma_{2}}\right) \frac{\left\langle k^{2}\right\rangle}{\langle k\rangle} \text {. }
$$

From the expression of $R_{0}$, we find that the first term $\left(\frac{\alpha \beta_{1}\left\langle k^{2}\right\rangle}{\left(m+\gamma_{1}\right)\langle k\rangle}\right)$ represents the number of individuals who are infected by an asymptomatically infected individual during recovery and latent period, and the third term $\left(\frac{(1-\alpha) \beta_{2}\left\langle k^{2}\right\rangle}{\gamma_{2}\langle k\rangle}\right)$ is the number of individuals who are infected by a symptomatically infected individual during the recovery period, whereas the second term $\left(\frac{m \alpha \beta_{2}\left\langle k^{2}\right\rangle}{\left(m+\gamma_{1}\right) \gamma_{2}\langle k\rangle}\right)$ indicates the number of individuals who are infected by the individual that is transformed from an asymptomatically infected to a symptomatical one.

Nowadays, the most attractive issue for the public is how to control the spread of COVID-19. Therefore, we are not going to elaborate the stability of system (1) here, with analysis being attached in "Appendix A". In the following sections, we focus on how to curb the spread of COVID-19 and analyse the outbreak based on the epidemic data of Wuhan from January 24 to March 2.

Throughout the paper, $S(t), A(t), I(t)$ and $R(t)$ are the fraction of susceptible, asymptomatically infected, symptomatically infected and removed individuals on network $G$, respectively, where $S(t)=\sum_{k} p(k) S_{k}(t)$, $A(t)=\sum_{k} p(k) A_{k}(t), I(t)=\sum_{k} p(k) I_{k}(t)$ and $R(t)=\sum_{k} p(k) R_{k}(t)$, and $S(t)+A(t)+I(t)+R(t)=$ 1. Owing to the truth that only the infected can turn into the removed, the removed in the stable state (referred to as $R_{\infty}$ ) represents the fraction of all infected indi- viduals from the outbreak to extinction of COVID-19. Furthermore, we refer to the measure of cutting off connections between symptomatically infected individuals and their neighbours, and that of cutting off connections between hub nodes and their neighbours as Measures 1 and 2, respectively.

\section{Numerical simulation}

Real-world networks are usually scale free [29]. Thus, we use the algorithm proposed by Barabási and Albert [30] to generate BA networks of size $N=1000$, and carry out simulations on these networks. Specifically, start with a fully connected network of $m_{0}=3$ nodes, then sequentially add the remaining nodes, each connecting to $m=3$ existing nodes with a probability which is proportional to the number of links that the existing nodes already have. Furthermore, because cutting off connections between symptomatically infected individuals and their neighbours (Measure 1) at each step is a dynamical process, we take the Monte Carlo (MC) method to obtain the values $S(t), A(t), I(t)$ and $R(t)$. Therefore, initially we set $S(t)=0.99, A(t)=0.005, \quad I(t)=0.005$ and $R(t)=0$, and seed the infected individuals on a BA network randomly. Then, we take 100 repetitions of MC simulations on the network, and average corresponding values over 10 BA networks. Since $S(t)+A(t)+I(t)+R(t)=1$, we only present the evolution of $A(t), I(t)$ and $R(t)$.

First, we explore the impact of recovery rate $\gamma_{1}$ and removed rate $\gamma_{2}$ on the spread of COVID-19. From Fig. 1, we discover that increasing $\gamma_{1}$ and $\gamma_{2}$ can obviously suppress the number of infected cases. Specifically, comparing Panels (a) and (b), we observe that $A(t)$ and $I(t)$ in Panel (a) are smaller than those in Panel (b). Similarly, $A(t)$ and $I(t)$ are much smaller in Panel (c) than those in Panel (b). These indicate that increasing the recovery rate and removed rate can effectively curb the spread of COVID-19.

Next, we investigate the roles of Measures 1 and 2 playing in the inhibition of COVID-19. We can obtain that taking Measures 1 and 2 immediately is the best approach to curb the spread of the pandemic. Specifically, when $\gamma_{1}=0$ and $\gamma_{2}=0.1$, without any measures taken to control the spread, the pandemic becomes prevalent (see Fig. 1a), and with measures taken, $R_{\infty}$ becomes much smaller (refer to the top panels of Fig. 


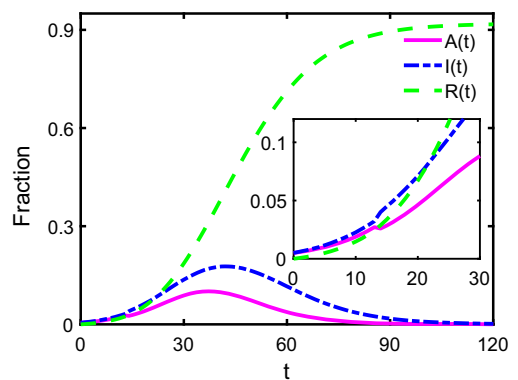

(a) $\gamma_{1}=0, \gamma_{2}=0.1$

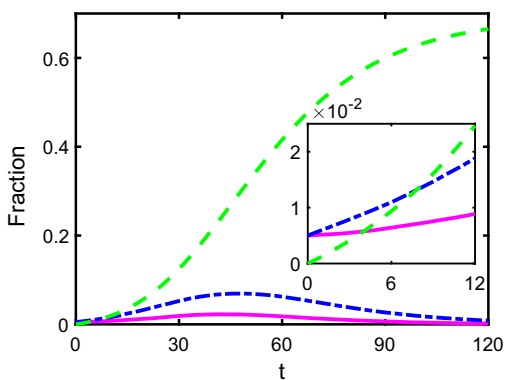

(b) $\gamma_{1}=0.1, \gamma_{2}=0.1$

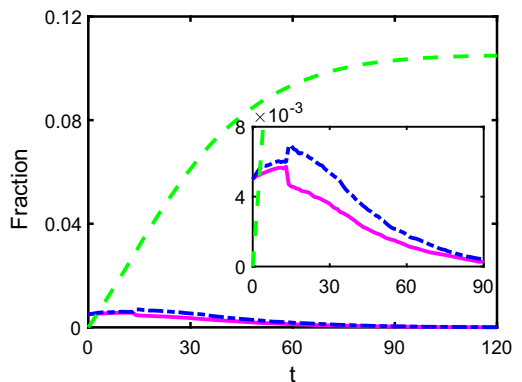

(c) $\gamma_{1}=0.1, \gamma_{2}=0.2$
Fig. 1 Time evolution of $A(t), I(t)$ and $R(t)$, where $\beta_{1}=$ $0.25, \beta_{2}=0.3, \alpha=0.3, \tau=14, m=0.9$. The pink solid line, blue dotted-dashed line and green dashed line represent the fraction of asymptomatically infected, symptomatically infected and removed individuals, respectively

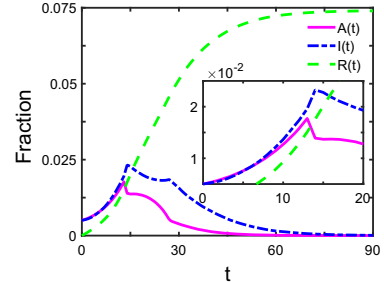

(a)

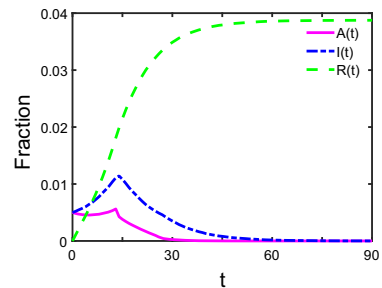

(e)

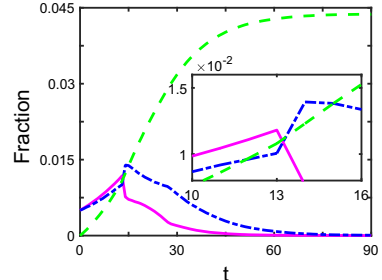

(b)

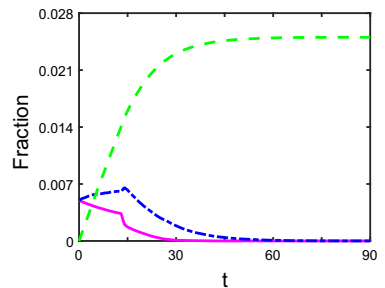

(f)

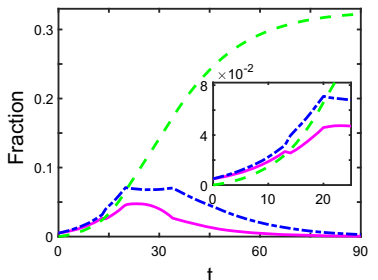

(c)

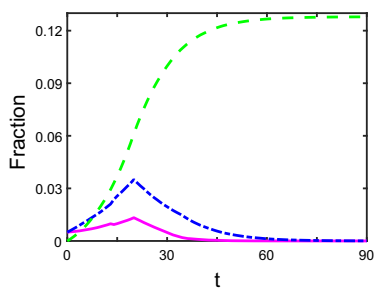

(g)

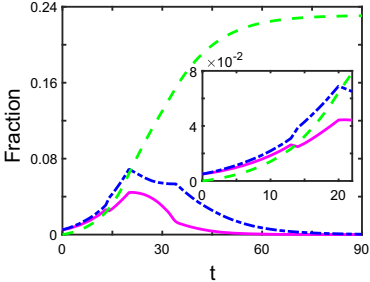

(d)

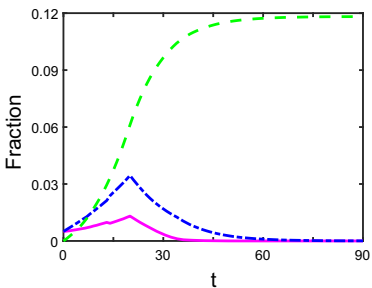

(h)

Fig. 2 Time evolution of $A(t), I(t)$ and $R(t)$, where $\beta_{1}=$ $0.25, \beta_{2}=0.3, \alpha=0.3, \tau=14$, and $m=0.9$. In the top panels, $\gamma_{1}=0$ and $\gamma_{2}=0.1$, and in the bottom panels, $\gamma_{1}=0.1$ and $\gamma_{2}=0.1$. a, e Measure 1 is taken immediately at $t=0 ; \mathbf{b}$, f Measures 1 and 2 are taken from $t=0 ; \mathbf{c}, \mathbf{g}$ Measure 1 comes

2), especially in the case of both Measures 1 and 2 taken. Furthermore, comparing with the case that measures are taken immediately (see Fig. 2a, b), we can observe that for the case of taking measures at $t=20$, $R_{\infty}$ is much larger and the time that $I(t)$ arrives at zero becomes longer (Fig. 2c, d). Similar observations can be obtained from the bottom panels of Fig. 2 for $\gamma_{1}=0.1$ and $\gamma_{2}=0.1$. These indicate that to curb the spread of COVID-19, we should take Measures 1 and 2 immediately, otherwise the COVID-19 would spread

into force at $t=20 ; \mathbf{d}, \mathbf{h}$ two measures come into force at $t=20$. We set that nodes with degree of over 10 are hub nodes. The pink solid line, blue dotted-dashed line and green dashed line represent the fraction of asymptomatically infected, symptomatically infected and removed individuals, respectively

rapidly around the globe and the duration time will become very long, which might cause serious consequence.

In summary, the best way to curb the spread of COVID-19 is to increase the recovery rate and the removed rate and cut off connections between symptomatically infected individuals and their neighbours, as well as to cut off connections between hub nodes and their neighbours. Indeed, the measures proposed by us coincide with those the government had taken to 
prevent the spread of COVID-19. Specifically, in order to prevent the spread of COVID-19, the government encourages the public to enhance immunity, segregates the infected with the symptomatic, prevents citizens from aggregating, by shutting down all the places of entertainment and imposing a home quarantine mechanism. Increasing the recovery rate and the removed rate means enhancing immunity. In fact, the most effective way to prevent the spread of the pandemic is vaccination (that is, $\gamma_{1}=\gamma_{2}=1$ ). However, since COVID19 is single-stranded RNA virus, vaccine development is difficult and faces many uncertainties. Thus, one of the feasible methods to curb the spread of COVID19 is to enhance individual immunity. Measure 1 represents quarantining the infected with symptoms and Measure 2 represents preventing citizens from aggregating. From Eq. (2), Measure 1 reduces not only $\frac{\left\langle k^{2}\right\rangle}{\langle k\rangle}$, but also $\beta_{1}$ and $\beta_{2}$. And Measure 2 weakens the effect of hub nodes to further reduce $\frac{\left\langle k^{2}\right\rangle}{\langle k\rangle}$.

In Fig. 2, the dynamical behaviours of system (1) are more complicated with $\gamma_{1}=0$ and $\gamma_{2}=0.1$, thus we explore the effect of network structures on the pandemic when $\gamma_{1}=0, \gamma_{2}=0.1$ and Measures 1 and 2 are taken immediately. From Fig. 3, we discover that the spread of the pandemic almost has the same tendency. Specifically, when measures are taken to control the spread immediately, at $t=14, I(t)$ reaches peak and $A(t)$ drops, then during $t=14$ to $28, I(t)$ falls slowly and $A(t)$ falls fast. When measures come into force at $t=20$, we can obtain that when the average degree of network is 4 or $6, I(t)$ and $A(t)$ reach peaks at $t=20$, then during the next latent period $I(t)$ falls slowly and $A(t)$ falls fast, finally after $t=34$ they all arrive at zero. The phenomenon indicate that the latent period and measures play important roles in the spread of the pandemic, which is beneficial for us to analyse the spread of COVID-19 by using the epidemic data of Wuhan from January 24 to March 2 in the next section. Furthermore, it is found that with the average degree of network changing from 2 to 4 and $6, R_{\infty}$ becomes larger and the duration of the outbreak becomes longer. Thus, another feasible way to curb the spread of COVID-19 is to reduce the density of social networks, such as restricting mobility and decreasing in-person social contacts.

\section{Data analysis}

In this section, we use the epidemic data of Wuhan from January 24 to March 2 to analyse the spread of COVID19 in the city after January 23 . We define the net increment of the infected with symptoms as $\Delta n I(t)$ at $t$. Obviously, $\triangle n I(t)=\Delta I(t)-\triangle R(t)$, where $\Delta I(t)$ is the increment of infected cases at $t$, and $\Delta R(t)$ is the increment of removed cases at $t$. According to the official data published by the Chinese government, we can obtain $\triangle n I$ from January 24 to March 2, as shown in Fig. 3a (the data are attached in "Appendix B"). Similarly, we define the net increment rate of symptomatically infected individuals as $\Delta n \operatorname{Ir}(t)$ at $t$, where $\triangle n I r(t+1)=I(t+1)-I(t)$.

From Fig. 4, we realize that the spread of the pandemic is sensitive to the latent period. Specifically, from Fig. 4b, c, we observe that at about $t=14$ (28), $\Delta n I r$ goes up sharply then falls, and for $t \in(1,13)((15,26))$, $\triangle n I r$ fluctuates. In Fig. 4a, we can obtain that from January 23 to February 4 (except January 27 and 28), $\triangle n I$ rose, then from February 4 to $13, \Delta n I$ fluctuated and after February 13, $\Delta n I$ had a downward trend.

Thus, in our opinion, the first stage of the outbreak of COVID-19 is from January 23 to February 4. In this stage, because there were many asymptomatically infected people (secondary infected) before January 23, $\Delta n I$ rose continually even though the government had taken efficient measures to prevent the spread of COVID-19 on January 23. In the second stage, from February 4 to $13, \Delta n I$ fluctuated because the asymptomatically infected people began to display symptoms. After February 13, as the third stage, the trend of $\Delta n I$ was down and especially $\triangle n I$ decreased to negative after February 19. This indicates that the spread of COVID-19 is gradually under control.

From the aforementioned analysis of Fig. 4, we realize that the net increment rate of symptomatically infected individuals $\triangle n I r$ fluctuates during the latent period. And throughout the spread of COVID-19 in Wuhan, the net increment of the infected with symptoms $\Delta n I$ fluctuated from February 4 to 13 , thus we reckon that the latent period is around 9 days. Thus, the opinion that the latent period can be extremely longer than one or two month is wrong. Perhaps there are some cases that asymptomatically infected people begin to exhibit symptoms after one month, but these are small probability events. 


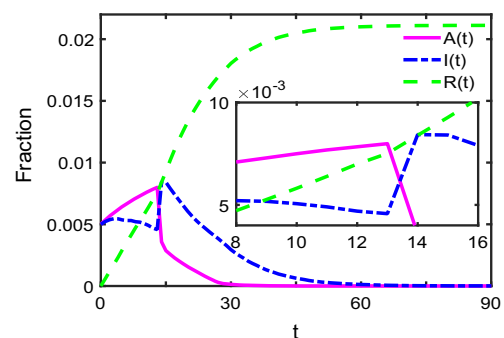

(a)

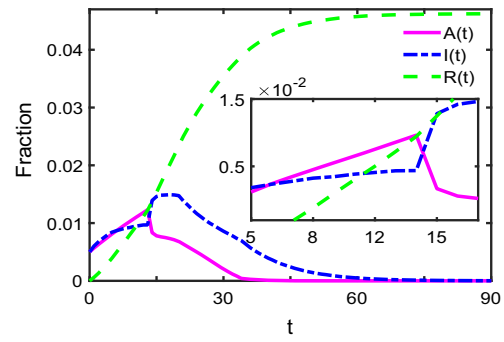

(d)

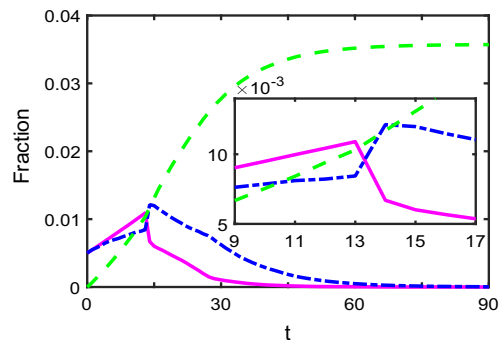

(b)

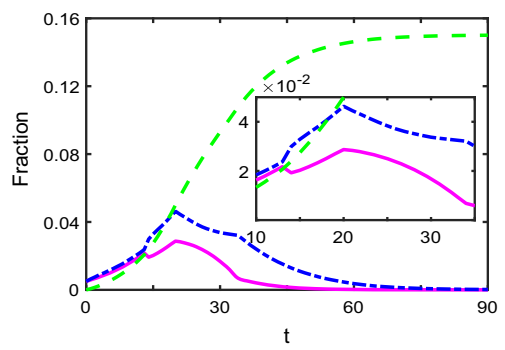

(e)

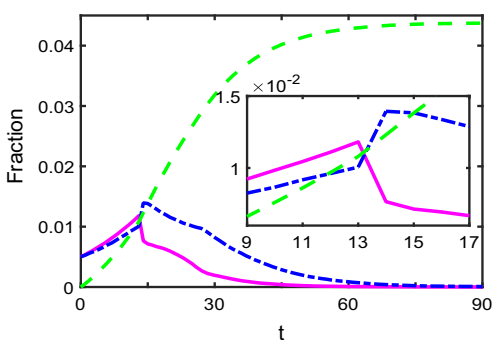

(c)

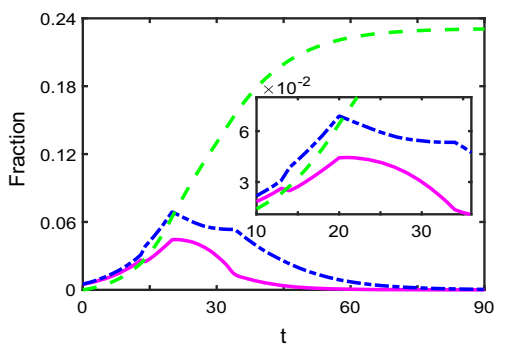

(f)
Fig. 3 Time evolution of $A(t)$ and $I(t)$, where $\beta_{1}=0.25, \beta_{2}=$ $0.3, \alpha=0.3, \tau=14, m=0.9, \gamma_{1}=0$ and $\gamma_{2}=0.1$. From left to right, the average degree of network is 2,4 , and 6 , respectively. In top panels, measures are taken immediately, whereas in the bottom panels, measures come into force at $t=20$. The pink solid line, blue dotted-dashed line and green dashed line represent the fraction of asymptomatically infected, symptomatically infected and removed individuals, respectively

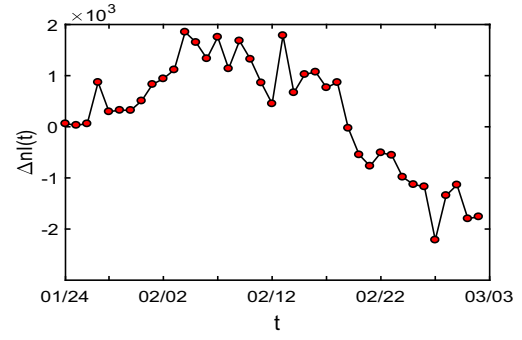

(a)

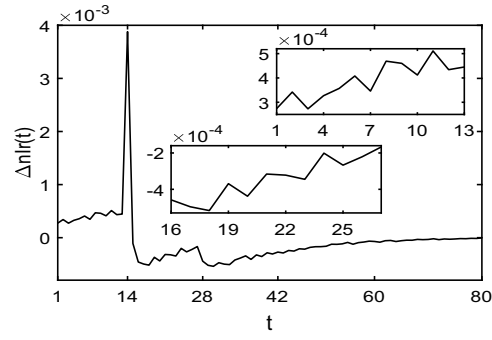

(b)

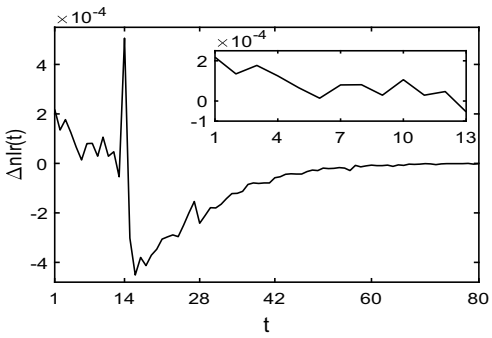

(c)
Fig. 4 a The net increment of the infected $\Delta n I$ from January 24 to March 2. b The net increment rate of the symptomatically infected $\triangle n I r$ with $\gamma_{1}=0$, and $\mathbf{c}$ the net increment rate

\section{Conclusion}

In this paper, we propose a SAIR model on social networks to model the spread of COVID-19 and analyse the outbreak based on the epidemic data of Wuhan from January 24 to March 2. By calculating the basic reproduction number and carrying out Monte Carlo simulations, we can obtain that to curb the spread of the pandemic, the measures proposed by us are in fair agree- of the symptomatically infected $\Delta n I r$ with $\gamma_{1}=0.1$. We set $\beta_{1}=0.25, \beta_{2}=0.3, \alpha=0.3, \tau=14, m=0.9$ and $\gamma_{2}=0.1$, and Measures 1 and 2 are taken immediately

ment with the measures the government had taken. In particular, we divide the outbreak of COVID-19 in Wuhan into three stages to explain why the infected cases increased though the government had taken efficient measures in the early stage. Furthermore, we find that with the average degree of network ascending, the pandemic would spread more rapidly and the duration time becomes longer. Therefore, another feasible method to curb the spread of the pandemic is to 
reduce the density of social networks, such as limiting the mobility and in-person social contacts.

Acknowledgements This work was supported in part by National Key Research and Development Program of China under Grant No. 2018AAA0101100, in part by the National Natural Science Foundation of China under grant no. 61973241, and in part by the Natural Science Foundation of Hubei Province No. 2019 CFA007.

\section{Compliance with ethical standards}

Conflict of interest No conflict to interest.

\section{Appendix A}

System $(1)$ has an equilibrium $\begin{gathered}E^{*} \\ =\left(S_{k_{1}}^{*}, \ldots, S_{k_{n}}^{*}, 0, \ldots, 0,1-S_{k_{1}}^{*}, \ldots, 1-S_{k_{n}}^{*}\right)^{\top} .\end{gathered}$ Linearizing system (1) at equilibrium $E^{*}$, we can obtain the following system

$$
\begin{aligned}
& \frac{\mathrm{d} X(t)}{\mathrm{d} t} \\
& =\left[\begin{array}{cccr}
0 & -\beta_{1} S^{*} P & -\beta_{2} S^{*} P & 0 \\
0 & \alpha \beta_{1} S^{*} P-\gamma_{1} I_{n} & \alpha \beta_{2} S^{*} P & 0 \\
0 & (1-\alpha) \beta_{1} S^{*} P & (1-\alpha) \beta_{2} S^{*} P-\gamma_{2} I_{n} & 0 \\
0 & \gamma_{1} I_{n} & \gamma_{2} I_{n} & 0
\end{array}\right] \\
& X(t)+\left[\begin{array}{cccc}
0 & 0 & 0 & 0 \\
0-m I_{n} & 0 & 0 \\
0 & m I_{n} & 0 & 0 \\
0 & 0 & 0 & 0
\end{array}\right] X(t-\tau),
\end{aligned}
$$

where $S^{*}=\operatorname{diag}\left(S_{k_{1}}^{*}, \ldots, S_{k_{n}}^{*}\right)$.

Then, the characteristic polynomial of the coefficient matrix of system (A.1) is
According to Schur's Lemma, there is a unitary matrix $U$ such that $S^{*} P=U^{-1} \bar{P} U$. Then, the above equation can be written as

$$
\begin{aligned}
f(\lambda)= & \left|\lambda^{2} I_{n}\right| \mid \lambda^{2} I_{n}+\left[\left(\gamma_{1}+\gamma_{2}\right) I_{n}-\left(\alpha \beta_{1}\right.\right. \\
+ & \left.\left.(1-\alpha) \beta_{2}\right) \bar{P}\right] \lambda+\left[\gamma_{1} \gamma_{2} I_{n}-\left(\alpha \beta_{1} \gamma_{2}\right.\right. \\
& \left.\left.+(1-\alpha) \beta_{2} \gamma_{1}\right) \bar{P}\right]+\left(\lambda I_{n}\right. \\
& \left.+\gamma_{2} I_{n}-\beta_{2} \bar{P}\right) m e^{-\lambda \tau} \mid \\
= & \lambda^{2 n}\left(\lambda+\gamma_{2}\right)^{n-1}\left(\lambda+\gamma_{1}+m e^{-\lambda \tau}\right)^{n-1}\left(\lambda^{2}\right. \\
& \left.+q_{1} \lambda+q_{2}+m\left(\lambda+q_{3}\right) e^{-\lambda \tau}\right),
\end{aligned}
$$

where $\bar{P}=\left[\begin{array}{cccc}K & \frac{k_{1} k_{2} p\left(k_{2}\right) S_{k_{1}}^{*}}{\langle k\rangle} & \ldots & \frac{k_{1} k_{n} p\left(k_{n}\right) S_{k_{1}}^{*}}{\langle k\rangle} \\ 0 & 0 & \ldots & 0 \\ \vdots & \vdots & \ddots & \vdots \\ 0 & 0 & \ldots & 0\end{array}\right], K=$ $\frac{\sum_{k} k^{2} p(k) S_{k}^{*}}{\langle k\rangle}, q_{1}=\gamma_{1}+\gamma_{2}-\left(\alpha \beta_{1}+(1-\alpha) \beta_{2}\right) K, q_{2}=$ $\gamma_{1} \gamma_{2}-\left(\alpha \beta_{1} \gamma_{2}+(1-\alpha) \beta_{2} \gamma_{1}\right) K$ and $q_{3}=\gamma_{2}-\beta_{2} K$.

Here, we present the following hypotheses that will be used:

$$
\begin{aligned}
& \left(H_{1}\right) \quad\left(q_{1}^{2}-2 q_{2}-m^{2}\right)^{2}-4\left(q_{2}^{2}-m^{2} q_{3}^{2}\right)>0, \\
& \left(H_{2}\right) q_{1}^{2}-2 q_{2}-m^{2} \geq 0, \text { and } q_{2}^{2}-m^{2} q_{1}<0, \\
& \left(H_{3}\right) q_{1}^{2}-2 q_{2}-m^{2}<0 .
\end{aligned}
$$

Proposition 1 (i) If $R_{0}<1, R_{1}<1, m<\gamma_{1}$, and Hypothesis $\left(H_{1}\right)$ does not hold, for any $\tau \geq 0$, the real part of all eigenvalues of Eq. (A.2) is less than or equal to zero.

$$
\begin{aligned}
& f(\lambda)=\left|\begin{array}{cccc}
\lambda I_{n} & \beta_{1} S^{*} P & \beta_{2} S^{*} P & 0 \\
0 & \lambda I_{n}-\alpha \beta_{1} S^{*} P+\gamma_{1} I_{n}+m e^{-\lambda \tau} I_{n} & -\alpha \beta_{2} S^{*} P & 0 \\
0 & -(1-\alpha) \beta_{1} S^{*} P-m e^{-\lambda \tau} I_{n} & \lambda I_{n}-(1-\alpha) \beta_{2} S^{*} P+\gamma_{2} I_{n} & 0 \\
0 & -\gamma_{1} I_{n} & -\gamma_{2} I_{n} & \lambda I_{n}
\end{array}\right| \\
& =\left|\lambda^{2} I_{n}\right| \mid \lambda^{2} I_{n}+\left[\left(\gamma_{1}+\gamma_{2}\right) I_{n}-\left(\alpha \beta_{1}+(1-\alpha) \beta_{2}\right) S^{*} P\right] \lambda+\left[\gamma_{1} \gamma_{2} I_{n}-\left(\alpha \beta_{1} \gamma_{2}\right.\right. \\
& \left.\left.+(1-\alpha) \beta_{2} \gamma_{1}\right) S^{*} P\right]+\left(\lambda I_{n}+\gamma_{2} I_{n}-\beta_{2} S^{*} P\right) m e^{-\lambda \tau} \mid \text {. }
\end{aligned}
$$


(ii) If $m>\gamma_{1}$, at $\tau_{1}^{j}=\frac{1}{\omega_{1, c}}\left(\arcsin \left(\frac{\omega_{1, c}}{m}\right)+\right.$ $2 j \pi), j=0,1, \ldots, E q$. (A.2) has a pair of pure imaginary roots $\pm \omega_{1, c} i$, where $\omega_{1, c}=\sqrt{m^{2}-\gamma_{1}^{2}}$.

(iii) if Hypotheses $\left(H_{1}\right)$ and $\left(H_{2}\right)$ or Hypotheses $\left(H_{1}\right)$ and $\left(H_{3}\right)$ hold, at $\tau_{2}^{j}=$ $\frac{1}{\omega_{2, c}}\left(\arcsin \left(\frac{m \omega_{2, c}\left(\omega_{2, c}^{2}-q_{2}+q_{3}\right)}{m^{2}\left(\omega_{2, c}^{2}+q_{3}^{2}\right)}\right)+2 j \pi\right), j=0,1, \ldots$, $E q$. (A.2) has a pair of pure imaginary roots $\pm \omega_{2, c} i$, where $\omega_{2, c}$ satisfies $\left(\omega^{2}\right)^{2}+\left(q_{1}-2 q_{2}-m^{2}\right) \omega^{2}+q_{2}^{2}-$ $m^{2} q_{3}^{2}=0$.

Proof (i) If $\tau=0$, Eq. (A.2) can be expressed as $f(\lambda)=\lambda^{2 n}\left(\lambda+\gamma_{2}\right)^{n-1}\left(\lambda+\gamma_{1}+m\right)^{n-1}\left(\lambda^{2}+\left(q_{1}+m\right) \lambda+q_{2}+m q_{3}\right)$.

According to Hurwitz Criterion, the real part of all eigenvalues of Eq. (A.2) is less than or equal to zero if and only if $q_{1}+m>0$ and $q_{2}+m q_{3}>0$, that is,

$$
\left\{\begin{array}{l}
\gamma_{1}+\gamma_{2}+m-\left(\alpha \beta_{1}+(1-\alpha) \beta_{2}\right) K \\
=\left(\gamma_{1}+\gamma_{2}+m\right)\left(1-R_{1}\right)>0, \\
\left(\gamma_{1}+m\right) \gamma_{2}-\left(m \beta_{2}+\alpha \beta_{1} \gamma_{2}+(1-\alpha) \beta_{2} \gamma_{1}\right) K \\
=\left(\gamma_{1}+m\right) \gamma_{2}\left(1-R_{2}\right)>0,
\end{array}\right.
$$

where $R_{1}=\frac{\alpha \beta_{1}+(1-\alpha) \beta_{2}}{\gamma_{1}+\gamma_{2}+m} \frac{\left\langle k^{2}\right\rangle}{\langle k\rangle}$ and $R_{2}=\left(\frac{\alpha \beta_{1}}{m+\gamma_{1}}+\right.$ $\left.\frac{m \alpha \beta_{2}}{\left(m+\gamma_{1}\right) \gamma_{2}}+\frac{(1-\alpha) \beta_{2}}{\gamma_{2}}\right) \frac{\sum_{k} k^{2} p(k) S_{k}^{*}}{\langle k\rangle}$.

Because $S_{k}(t) \leq 1$, there is $R_{2}<R_{0}$. Thus, if $R_{0}<$ 1 and $R_{1}<1$, the real part of all eigenvalues of Eq. (A.2) is non-positive.

(ii) Suppose that system (A.1) has a pair of pure imaginary eigenvalues $\pm \omega i(\omega \neq 0)$. Obviously, $\lambda$ satisfies $f_{1}(\lambda)=\lambda+\gamma_{1}+m e^{-\lambda \tau}=0$ or $f_{2}(\lambda)=$ $\lambda^{2}+q_{1} \lambda+q_{2}+m\left(\lambda+q_{3}\right) e^{-\lambda \tau}$. For simplicity, we set $\lambda=\omega i$. In the following, we discuss these two cases separately.

Case 1: $\lambda$ satisfies $f_{1}(\lambda)=\lambda+\gamma_{1}+m e^{-\lambda \tau}=0$.

Substituting $\lambda=\omega i$ and $e^{\omega \tau i}=\cos (\omega \tau)+i \sin (\omega \tau)$ into equation $f_{1}(\lambda)=0$, we obtain

$\omega i+\gamma_{1}+m\left(\cos \left(\omega_{1} \tau\right)-i \sin \left(\omega_{1} \tau\right)\right)=0$.

Separating the real part from the imaginary part, we can write Eq. (A.3) as

$\left\{\begin{array}{l}\gamma_{1}+m \cos (\omega \tau)=0 \\ \omega-m \sin (\omega \tau)=0\end{array}\right.$
Combining with $\sin (\omega \tau)^{2}+\cos (\omega \tau)^{2}=1$, we obtain $\omega^{2}=m^{2}-\gamma_{1}^{2}$. Thus, if $m>\gamma_{1}$, there is $\omega=\sqrt{m^{2}-\gamma_{1}^{2}}$ and the corresponding $\tau$ is $\tau_{1}^{j}=\frac{1}{\sqrt{m^{2}-\gamma_{1}^{2}}}\left(\arcsin \left(\frac{\sqrt{m^{2}-\gamma_{1}^{2}}}{m}\right)+2 j \pi\right), j=0,1, \ldots$, whereas if $m<\gamma_{1}$, a real $\omega$ does not exist.

Case 2: $\lambda$ satisfies $f_{2}(\lambda)=\lambda^{2}+q_{1} \lambda+q_{2}+m(\lambda+$ $\left.q_{3}\right) e^{-\lambda \tau}$.

Similarly, we obtain

$$
\left\{\begin{array}{l}
-\omega^{2}+q_{2}+m \omega \sin (\omega \tau)+m q_{3} \cos (\omega \tau)=0 \\
q_{1} \omega+m \omega \cos (\omega \tau)-m q_{3} \sin (\omega \tau)=0
\end{array}\right.
$$

Then, $\omega$ satisfies $\left(\omega^{2}\right)^{2}+\left(q_{1}-2 q_{2}-m^{2}\right) \omega^{2}+q_{2}^{2}-$ $m^{2} q_{3}^{2}=0$. Thus, if Hypotheses $\left(H_{1}\right)$ and $\left(H_{2}\right)$ or Hypotheses $\left(H_{1}\right)$ and $\left(H_{3}\right)$ hold, we can get $\omega_{2, c}$ and $\tau_{2}^{j}$.

Proposition 2 (i) If $m>\gamma_{1}$, the transversality condition of $f_{1}(\lambda)=0$ is

$$
\left.\left(\frac{\mathrm{d}(\operatorname{Re} \lambda)}{\mathrm{d} \tau}\right)^{-1}\right|_{\tau=\tau_{1}^{j}}>0, j=0,1, \ldots
$$

(ii) if Hypotheses $\left(H_{1}\right)$ and $\left(H_{2}\right)$ or Hypotheses $\left(H_{1}\right)$ and $\left(H_{3}\right)$ hold, the transversality condition of $f_{2}(\lambda)=$ 0 is

$$
\left.\left(\frac{\mathrm{d}(\operatorname{Re} \lambda)}{\mathrm{d} \tau}\right)^{-1}\right|_{\tau=\tau_{2}^{j}}>0, j=0,1, \ldots
$$

Proof (i) Taking the derivative of $\lambda$ with respect to $\tau$, we get

$\left(1-m \tau e^{-\lambda \tau}\right) \frac{\mathrm{d} \lambda}{\mathrm{d} \tau}-m \lambda e^{-\lambda \tau}=0$,

that is,

$\left(\frac{\mathrm{d} \lambda}{\mathrm{d} \tau}\right)^{-1}=\frac{1-m \tau e^{-\lambda \tau}}{m \lambda e^{-\lambda \tau}}=-\frac{1}{\lambda\left(\lambda+\gamma_{1}\right)}-\frac{\tau}{\lambda}$.

Therefore, the transversality condition of $f_{1}(\lambda)=0$ is

$\left.\left(\frac{\mathrm{d}(\operatorname{Re} \lambda)}{\mathrm{d} \tau}\right)^{-1}\right|_{\tau=\tau^{j}}=\frac{1}{m^{2}}>0$. 
(ii) By the same method, we can prove $\left.\left(\frac{d(\operatorname{Re} \lambda)}{d \tau}\right)^{-1}\right|_{\tau=\tau_{2}^{j}}>$ $0, j=0,1, \ldots$

From Propositions 1 and 2, we can obtain the following theorem.

Theorem 1 (i) If $R_{0}>1$, for any $\tau \geq 0$, the equilibrium $E^{*}$ of system (1) is unstable.

(ii) If $R_{0}<1, R_{1}<1, m<\gamma_{1}$ and Hypothesis $\left(H_{1}\right)$ does not hold, then for any $\tau \geq 0$, system (1) will get stabilized on the equilibrium $E^{*}$.

(iii) Suppose $R_{0}<1, R_{1}<1, m>\gamma_{1}$ and Hypothesis $\left(H_{1}\right)$ does not hold. If $\tau \in\left[0, \tau_{1}^{0}\right)$, the equilibrium $E^{*}$ of system (1) is stable. If $\tau>\tau_{1}^{0}$, the equilibrium $E^{*}$ of system (1) will be unstable. Furthermore, when $\tau=\tau_{1}^{j}, j=0,1, \ldots$, Hopf bifurcation occurs.

(iv) Suppose $R_{0}<1, R_{1}<1, m<\gamma_{1}$ and Hypotheses $\left(H_{1}\right)$ and $\left(H_{2}\right)$ or Hypotheses $\left(H_{1}\right)$ and $\left(H_{3}\right)$ hold. If $\tau \in\left[0, \tau_{2}^{0}\right)$, the equilibrium $E^{*}$ of system
(1) is stable. If $\tau>\tau_{2}^{0}$, the equilibrium $E^{*}$ of system (1) will be unstable. Furthermore, if $\tau=\tau_{2}^{j}$, Hopf bifurcation occurs.

(v) Suppose $R_{0}<1, R_{1}<1, m>\gamma_{1}$ and Hypotheses $\left(H_{1}\right)$ and $\left(H_{2}\right)$ or Hypotheses $\left(H_{1}\right)$ and $\left(H_{3}\right)$ hold. If $\tau \in\left[0, \min \left(\tau_{1}^{0}, \tau_{2}^{0}\right)\right)$, the equilibrium $E^{*}$ of system (1) is stable. If $\tau>\min \left(\tau_{1}^{0}, \tau_{2}^{0}\right)$, the equilibrium $E^{*}$ of system (1) will be unstable. Furthermore, when $\tau=\tau_{1}^{j}\left(\tau_{2}^{j}\right)$, Hopf bifurcation occurs.

\section{Appendix B}

According to the official data published by the Chinese government, we can obtain $\triangle I, \triangle R$ and $\triangle n I$ from January 24 to March 2, shown as Table 2.

Table 2 The epidemic data of Wuhan from January 23 to March 2, 2020

\begin{tabular}{|c|c|c|c|c|c|c|c|}
\hline Date & $\triangle I$ & $\Delta R^{\mathrm{a}}$ & $\triangle n I$ & Date & $\triangle I$ & $\Delta R^{\mathrm{a}}$ & $\triangle n I$ \\
\hline $1 / 24$ & 77 & $16(15+1)$ & 61 & $1 / 25$ & 46 & $15(7+8)$ & 31 \\
\hline $1 / 26$ & 80 & $20(18+2)$ & 60 & $1 / 27$ & 892 & $22(22)$ & 870 \\
\hline $1 / 28$ & 315 & $19(19)$ & 296 & $1 / 29$ & 356 & $32(25+7)$ & 324 \\
\hline $1 / 30$ & 378 & $56(30+26)$ & 322 & $1 / 31$ & 576 & $69(33+36)$ & 507 \\
\hline $2 / 1$ & 894 & $64(32+32)$ & 830 & $2 / 2$ & 1033 & $94(41+53)$ & 940 \\
\hline $2 / 3$ & 1242 & $127(48+79)$ & 1115 & $2 / 4$ & 1967 & $114(49+65)$ & 1853 \\
\hline $2 / 5$ & 1766 & $115(52+63)$ & 1651 & $2 / 6$ & 1501 & $167(64+103)$ & 1334 \\
\hline $2 / 7$ & 1985 & $231(67+164)$ & 1754 & $2 / 8$ & 1379 & $242(63+179)$ & 1137 \\
\hline $2 / 9$ & 1921 & $240(73+167)$ & 1681 & $2 / 10$ & 1552 & $229(67+162)$ & 1323 \\
\hline $2 / 11$ & 1104 & $243(72+171)$ & 861 & $2 / 12$ & $1072^{\mathrm{b}}$ & $620\left(82^{\mathrm{b}}+538\right)$ & 452 \\
\hline $2 / 13$ & $2243^{b}$ & $458(88+370)$ & 1785 & $2 / 14$ & $1001^{\mathrm{b}}$ & $332\left(77^{\mathrm{b}}+255^{\mathrm{b}}\right)$ & 669 \\
\hline $2 / 15$ & 1548 & $523(110+413)$ & 1025 & $2 / 16$ & 1690 & $619(76+543)$ & 1071 \\
\hline $2 / 17$ & 1600 & $833(72+761)$ & 767 & $2 / 18$ & 1660 & $792(116+676)$ & 868 \\
\hline $2 / 19$ & 615 & $641(88+553)$ & -26 & $2 / 20$ & 319 & $865(99+766)$ & -546 \\
\hline $2 / 21$ & 314 & $1082(90+992)$ & -768 & $2 / 22$ & 541 & $1047(82+965)$ & -506 \\
\hline $2 / 23$ & 348 & $903(131+772)$ & -555 & $2 / 24$ & 464 & $1447(56+1391)$ & -983 \\
\hline $2 / 25$ & 370 & $1498(42+1456)$ & -1128 & $2 / 26$ & 383 & $1554(19+1535)$ & -1171 \\
\hline $2 / 27$ & 313 & $2526(28+2498)$ & -2213 & $2 / 28$ & 420 & $1763(37+1726)$ & -1343 \\
\hline $2 / 29$ & 565 & $1701(26+1675)$ & -1136 & $3 / 1$ & 193 & $1990(32+1958)$ & -1797 \\
\hline $3 / 2$ & 111 & $1870(24+1846)$ & -1759 & & & & \\
\hline
\end{tabular}

http://www.hubei.gov.cn/zhuanti/2020/gzxxgzbd/zxtb/index_16.shtml

${ }^{\text {a }}$ Here $\triangle R$ is the sum of the increment of dead cases and recuperative cases

$\mathrm{b}$ The native data includes clinical diagnosis, and the data has been processed. $1072=13436-12364,82=216-134,2243=$ $3910-(14031-12364), 1001=1923-922,77=107-30,255=486-231$ 


\section{References}

1. Peiris, J.S.M., Lai, S.T., Poon, L.I.M., et al.: Coronavirus as a possible cause of severe acute respiratory syndrome. Lancet 361(9366), 1319-1325 (2003)

2. Assiri, A., McGeer, A., Perl, T.M., et al.: Hospital outbreak of middle east respiratory syndrome coronavirus. N. Engl. J. Med. 369(5), 407-416 (2013)

3. Assiri, A., Al-Tawfiq, J.A., Al-Rabeeah, A.A., et al.: Epidemiological, demographic, and clinical characteristics of 47 cases of middle east respiratory syndrome coronavirus disease from Saudi Arabia: a descriptive study. Lancet Infect. Dis. 13(9), 752-761 (2013)

4. Huang, C., Wang, Y., Li, X., et al.: Clinical features of patients infected with 2019 novel coronavirus in Wuhan, China. Lancet 395(10223), 497-506 (2020)

5. Chen, N., Zhong, M., Dong, X., et al.: Epidemiological and clinical characteristics of 99 cases of 2019 novel coronavirus pneumonia in Wuhan, China: a descriptive study. Lancet 395(10223), 507-513 (2020)

6. Zhao, S., Zhuang, Z., Ran, J., et al.: The association between domestic train transportation and novel coronavirus (2019nCoV) outbreak in China from 2019 to 2020: a data-driven correlational report. Travel Med. Infect. Dis. 33, 101568 (2020)

7. Li, Q., Guan, X., Wu, P., et al.: Early transmission dynamics in Wuhan, China, of novel coronavirus-infected pneumonia. N. Engl. J. Med. 382(13), 1199-1207 (2010)

8. Hui, D.S., Azhar, E.I., Madani, T.A., et al.: The continuing 2019-nCoV epidemic threat of novel coronaviruses to global health-The latest 2019 novel coronavirus outbreak in Wuhan, China. Int. J. Infect. Dis. 91, 264-266 (2020)

9. Nishiura, H., Jung, S.M., Linton, N.M., et al.: The extent of transmission of novel coronavirus in Wuhan, China, 2020. J. Clin. Med. 9(2), 330 (2020)

10. Wang, P., Lu, J., Jin, Y., et al.: Statistical and network analysis of 1212 COVID-19 patients in Henan, China. Int. J. Infect. Dis. 95, 391-398 (2020)

11. Tang, B., Wang, X., Li, Q., et al.: Estimation of the transmission risk of 2019-nCov and its implication for public health interventions. J. Clin. Med. 9(2), 462 (2020)

12. Zhao, S., Lin, Q., Ran, J., et al.: Preliminary estimation of the basic reproduction number of novel coronavirus (2019nCoV) in China, from 2019 to 2020: a data-driven analysis in the early phase of the outbreak. Int. J. Infect. Dis. 92, 214-217 (2020)

13. Wu, J.T., Leung, K., Leung, G.M.: Nowcasting and forecasting the potential domestic and international spread of the 2019-nCoV outbreak originating in Wuhan, China: a modelling study. Lancet 395(10225), 689-697 (2020)

14. Wang, Y., Cao, J., Li, X., et al.: Edge-based epidemic dynamics with multiple routes of transmission on random networks. Nonlinear Dyn. 91(1), 403-420 (2018)
15. Liu, J., Wu, X., Lü, J., et al.: Infection-probability-dependent interlayer interaction propagation processes in multiplex networks. IEEE Trans. Syst. Man Cybernet. Syst. (2019). https://doi.org/10.1109/TSMC.2018.2884894

16. Gross, T., D’Lima, C.J.D., Blasius, B.: Epidemic dynamics on an adaptive network. Phys. Rev. Lett. 96(20), 208701 (2006)

17. Shao, Q., Xia, C., Wang, L., et al.: A new propagation model coupling the offline and online social networks. Nonlinear Dyn. 98(3), 2171-2183 (2019)

18. Xu, S., Wang, P., Zhang, C., et al.: Spectral learning algorithm reveals propagation capability of complex network. IEEE Trans. Cybernet. 49(2), 4253-4261 (2019)

19. Newman, M.E.J.: Spread of epidemic disease on networks. Phys. Rev. E 66(1), 016128 (2002)

20. Pastor-Satorras, R., Vespignani, A.: Epidemics and Immunization in Scale-Free Networks. Handbook of Graphs and Networks. Wiley, New York (2003)

21. Granell, C., Gómez, S., Arenas, A.: Dynamical interplay between awareness and epidemic spreading in multiplex networks. Phys. Rev. Lett. 111, 128701 (2013)

22. Wei, X., Wu, X., Chen, S., et al.: Cooperative epidemic spreading on a two-layered interconnected network. SIAM J. Appl. Dyn. Syst. 17(2), 1503-1520 (2018)

23. Zhang, Z., Liu, C., Zhan, X., et al.: Dynamics of information diffusion and its applications on complex networks. Phys. Rep. 51, 1-34 (2016)

24. Li, Y., Wu, X., Lu, J., et al.: Synchronizability of duplex networks. IEEE Trans. Circuits Syst. II-Express Briefs 63(2), 206-210 (2016)

25. Boccaletti, G., Bianconi, G., Criado, R., et al.: The structure and dynamics of multilayer networks. Phys. Rep. 544, 1-22 (2014)

26. Mei, G., Wu, X., Wang, Y., et al.: Compressive-sensingbased structure identification for multilayer networks. IEEE Trans. Cybernet. 48(2), 754-764 (2018)

27. Keeling, M.J., Eames, K.T.D.: Networks and epidemic models. J. R. Soc. Interface 2(4), 295-307 (2005)

28. van den Driessche, P., Watmough, J.: Reproduction numbers and sub-threshold endemic equilibria for compartmental models of disease transmission. Math. Biosci. 180, 29-48 (2002)

29. Albert, R., Barabasi, A.L.: Statistical mechanics of complex networks. Rev. Mod. Phys. 74(1), 47-97 (2002)

30. Barabási, A.-L., Albert, R.: Emergence of scaling in random networks. Science 286(5439), 509-512 (1999)

Publisher's Note Springer Nature remains neutral with regard to jurisdictional claims in published maps and institutional affiliations. 